to encourage fair and equitable mental healthcare budgetary provision and the development of national mental health policies, including mental health legislation, with effective national implementation programmes in low- and middleincome countries. Otherwise, vulnerable patients with mental disorders will continue to suffer in silence, without the protection of their human and civil rights to receive mental healthcare free of discrimination, ill-treatment and abuse. This challenge has recently been taken up by the Lancet, which launched a new movement for mental health (Horton, 2007), supported by a series of outstanding articles (e.g. Jacob et al, 2007; Saxena et al, 2007; Patel et al, 2007; Saraceno et al, 2007).

The current findings also suggest further avenues for research. Does the mere presence of mental health legislation ensure protection of the rights of patients? Is mental health legislation implemented correctly? Is the legislation followed and monitored adequately? Evidence gathered by the Mental Health Act Commission in England and Wales suggests that without constant vigilance by the state, mental health service providers fail to implement legislation appropriately (Mental Health Act Commission, 2006, 2008). This may also be true in other countries; clearer understanding of the way legislation is implemented would be of assistance to countries with and without adequate legislation.

\section{References}

Horton, R. (2007) Launching a new movement for mental health. Lancet, 370, 806.

Jacob, K. S., Sharan, P., Mirza, I., et al (2007) Global mental health 4. Mental health systems in countries: where are we now? Lancet, 370, 1061-1077.

Mental Health Act Commission (2006) Eleventh Biennial Report. Placed Amongst Strangers, paras 3.73-3.86. TSO (The Stationery Office).

Mental Health Act Commission (2008) Twelfth Biennial Report. Risk, Right and Recovery, ch. 8. TSO (The Stationery Office).

Patel, V., Araya, R., Chatterjee, S., et al (2007) Treatment and prevention of mental disorders in low and middle-income countries. Lancet, 370, 991-1005

Saraceno, B., van Ommeren, M., Batniji, R., et al (2007) Global mental health 5. Barriers to improvement of mental health services in lowincome and middle-income countries. Lancet, 370, 1164-1174.

Saxena, S., Thornicroft, G., Knapp, K., et al (2007) Global mental health 2. Resources for mental health: scarcity, inequity, and inefficiency. Lancet, 370, 878-889.

Shah, A. K. (2007) The importance of socio-economic status of countries for mental disorders in old age: a development of an epidemiological transition model. International Psychogeriatrics, 19, 785-787.

Shah, A. K. \& Bhat, R. (2008) Are elderly suicide rates improved by increased provision of mental health service resources? A cross-national study. International Psychogeriatrics, 17 March, 1-8.

World Health Organization (2001) World Health Report 2001 - Mental Health: New Understanding, New Hope. WHO.

World Health Organization (2004) Mental Health Policy, Plans and Programme. WHO.

\title{
Comparison of risperidone, olanzapine and quetiapine: effects on body weight, serum blood glucose and prolactin
}

\section{Haroon Rashid Chaudhry, ${ }^{1}$ Nadia Arshad, ${ }^{2}$ Saima Niaz, ${ }^{3}$ Tahir Suleman ${ }^{4}$ and Khalid A. Mufti ${ }^{5}$}

'1Head, Department of Psychiatry, Fatima Jinnah Medical College, Lahore, Pakistan, email pprc@wol.net.pk ${ }^{2}$ Deputy Manager, Research, College of Physicians and Surgeons Pakistan

${ }^{3}$ Trust Grade (Adult Psychiatry), Plymouth Teaching Primary Care Trust, Mount Gould Hospital, Plymouth, UK ${ }^{4}$ Consultant Psychiatrist (Working Age), Cumbria Partnership NHS Foundation Trust, Carlisle, UK

${ }^{5}$ Chairman, Horizon, Peshawar, Pakistan

\begin{abstract}
Chizophrenia is a chronic illness with a lifetime prevalence of $1 \%$ and with serious physical, social and economic consequences. Over the past decade, atypical antipsychotic medications have become the first-line treatment for schizophrenia (Breier et al, 2005).

The extensive use of atypical antipsychotics is based on their clinical efficacy (for both positive and negative symptoms) and lesser side-effects (e.g. extrapyramidal symptoms) compared with conventional antipsychotics. However, the unique pharmacodynamic profiles and accumulating evidence suggest that these agents, particularly olanzapine and risperidone, do have certain side-effects, including weight gain and elevated blood glucose and serum
\end{abstract}

prolactin levels. These side-effects are a burden to patients and may affect adherence to treatment. The prescribing clinician has to weigh up the risks and benefits of a particular antipsychotic in an individual case.

There is a growing concern about the metabolic syndrome and its complications with the long-term use of at least some of the atypical drugs (American Diabetes Association, 2004). Weight gain, high levels of cholesterol and high blood glucose concentrations are part of the metabolic syndrome. These factors increase the risk for diabetes mellitus and are a risk factor for coronary heart disease (Straker et al, 2005). Before the introduction of atypical antipsychotics, prolactin elevation was an inevitable risk of treatment with antipsychotics. 
Prolactin elevation is less of a concern with some of the atypical agents. The exception is risperidone, which results in prolactin elevations that are similar to those associated with first-generation antipsychotics (Allison et al, 1999).

The present study was designed to determine whether three atypical antipsychotics, risperidone, olanzapine and quetiapine, differ from each other in their effects on body weight and blood glucose and serum prolactin levels.

\section{Methods}

This randomised clinical trial was conducted at the outpatient Department of Psychiatry, Sir Ganga Ram Hospital and the Free Psychiatric Clinic at Ahbab Hospital, Ravi Road, Lahore, Pakistan. The study protocol was carried out in accordance with the Declaration of Helsinki.

One hundred and twenty drug-naive patients aged between 18 and 58 years who had schizophrenia, diagnosed according to DSM-IV-TR criteria, were eligible after they had given their informed consent. Patients with a total score on the Positive and Negative Symptoms Scale (PANSS) of 60 or more were included. Those with serious physical or neurological illnesses were excluded.

Patients were randomly allocated to receive risperidone $(n=40)$, olanzapine $(n=40)$ or quetiapine $(n=40)$. Dosing was flexible: risperidone, 1-6 mg/day; olanzapine, 5-20 $\mathrm{mg} /$ day; and quetiapine, 100-600 mg/day. Doses were adjusted for each patient to achieve maximum efficacy with minimum adverse effects. Each drug was administered twice daily for up to 90 days. The only concomitant medication allowed during the study period was lorazepam $(\leq 4 \mathrm{mg} /$ day).

Age, gender, marital status, education and family history of psychiatric disorder were recorded for each patient. Duration of illness ranged from 6 months to 4 years. Patients' body weight, blood glucose and serum prolactin levels were recorded at baseline, and at 15,30,60 and 90 days of taking antipsychotic medication. Blood was obtained approximately 12 hours after the last dose. Assessments based on the PANSS were conducted at baseline and 90 days after random assignment to treatment.

\section{Results}

The sample comprised 83 men (69\%) and 37 women (31\%). Their mean (s.d.) age was 35.4 (9.6) years and their mean (s.d.) duration of illness was 24.9 (11.3) months. Sixty-one patients (51\%) reported a family history of psychiatric illness. Mean (s.d.) doses of drugs were: 4.2 (1.1) mg risperidone, 16.9 (3.7) mg olanzapine and 342.8 (135.9) mg quetiapine. There were seven drop-outs in the risperidone group, three in the olanzapine group and one in the quetiapine group.

Olanzapine-treated patients had a significant increase in body weight and blood glucose level compared with those on risperidone or quetiapine. Risperidone-treated patients had a raised serum prolactin level compared with those on olanzapine or quetiapine. The results are given in Table 1.

The groups were matched at baseline. After 90 days, a significant decrease in PANSS score was observed in the risperidone group, to a mean of 35.7 (6.4), followed by the
Table 1 Side-effect profiles of the three atypical drugs

$\begin{array}{llcr} & \text { Stage } & \text { Mean } & \text { s.d. } \\ \text { Risperidone }(n=33) & & \\ \text { Body weight }(\mathrm{kg}) & \text { Baseline } & 69.0 & 9.5 \\ & 90 \text { days } & 70.7^{*} & 9.5 \\ \text { Blood glucose }(\mathrm{mg} / \mathrm{dl}) & \text { Baseline } & 114.9 & 27.2 \\ & 90 \text { days } & 118.0 & 19.1 \\ \text { Serum prolactin }(\mathrm{ng} / \mathrm{dl}) & \text { Baseline } & 16.6 & 8.3 \\ & 90 \text { days } & 49.3^{*} & 24.5 \\ & & & \\ \text { Olanzapine }(n=37) & & & \\ \text { Body weight }(\mathrm{kg}) & \text { Baseline } & 70.1 & 8.5 \\ & 90 \text { days } & 72.4^{*} & 8.6 \\ \text { Blood glucose }(\mathrm{mg} / \mathrm{dl}) & \text { Baseline } & 123.3 & 29.9 \\ & 90 \text { days } & 166.6^{*} & 53.2 \\ \text { Serum prolactin }(\mathrm{ng} / \mathrm{dl}) & \text { Baseline } & 14.0 & 6.0 \\ & 90 \text { days } & 14.9^{*} & 6.0 \\ & & & \\ \text { Quetiapine }(n=39) & & & \\ \text { Body weight }(\mathrm{kg}) & \text { Baseline } & 64.7 & 8.7 \\ & 90 \text { days } & 65.4^{*} & 8.7 \\ \text { Blood glucose }(\mathrm{mg} / \mathrm{dl}) & \text { Baseline } & 114.4 & 19.3 \\ & 90 \text { days } & 115.0 & 18.6 \\ \text { Serum prolactin }(\mathrm{ng} / \mathrm{dl}) & \text { Baseline } & 12.6 & 6.4 \\ & 90 \text { days } & 13.5^{*} & 6.6 \\ & & \end{array}$

${ }^{*} P<0.05$ for inter-group comparisons (see text).

olanzapine group, to a mean of 41.5 (6.3), and quetiapine group, to a mean of 41.9 (5.7) (Table 2).

\section{Discussion}

In a systematic review (Taylor \& McAskill, 2000), it was found that the risk of increase in body weight associated with olanzapine, quetiapine and risperidone was $14-27 \%$ at $6-8$ weeks and as high as $40 \%$ by 3.5 years. But there was a lack of direct long-term comparisons. However, Bryden \& Kopala (1999) suggest that the risk of weight gain is greatest with olanzapine, probably intermediate with risperidone and low with quetiapine, which is similar to the current study.

In a study by Gupta et al (2004) it was notable that weight declined when patients were switched from olanzapine to quetiapine after they had gained more than 20\% of their weight during olanzapine treatment. In that 10 -week study, 12 patients lost a mean of $2.25 \mathrm{~kg}$.

A trial comparing clozapine, olanzapine, risperidone and haloperidol found similar results as the current study for olanzapine and risperidone regarding elevation of blood glucose levels. Clozapine and haloperidol were associated with significantly elevated mean glucose levels after 8 weeks of treatment compared with risperidone. Changes in glucose levels were independent of weight increase in all four treatment groups; significant weight gain was observed in olanzapine-treated patients, followed by clozapine- and risperidone-treated patients (Gupta et al, 2004).

Table 2 PANSS scores in the three groups

\begin{tabular}{llllll}
\multirow{2}{*}{ Groups } & \multicolumn{3}{l}{ Baseline } & & \multicolumn{3}{l}{90 days } \\
\cline { 2 - 3 } Quean & Mean & s.d. & & Mean & s.d. \\
Risperidone $(n=39)$ & 61.0 & 4.1 & & 41.9 & 5.7 \\
Olanzapine $(n=37)$ & 66.2 & 5.3 & & 35.7 & 6.5 \\
& 64.6 & 4.8 & & 41.5 & 6.3
\end{tabular}

$P<0.05$ for both the decrease over time in all three groups. 
The current study found that risperidone gave a greater overall reduction of scores on PANSS at 90 days than did either olanzapine or quetiapine (see also Lindenmayer et al, 2003).

A double-blind comparison of olanzapine and quetiapine in the treatment of patients with schizophrenia and schizoaffective disorder showed that both medications improved symptoms, with similar response rates. Olanzapine appeared to be better than quetiapine on overall PANSS response, while quetiapine was significantly better than olanzapine on the disorientation scale only. In terms of side-effects, quetiapine appeared to have a lesser effect on prolactin levels. Both medications caused weight gain (Svesta et al, 2003).

Another study has compared olanzapine with risperidone in relation to serum prolactin levels. A significantly lower proportion of patients receiving olanzapine experienced an elevation above standard reference ranges in prolactin concentration (51.2\% v. 94.4\%) (Tran et al, 1997). The present study found a similar trend.

One study showed that both olanzapine and risperidone were effective, but with olanzapine apparently significantly more effective. Both medications were associated with a $2 \mathrm{~kg}$ weight gain during the course of the study (Svesta et al, 2003). These results are in line with those of the current study.

The current study also found at 90 days a $46 \%$ reduction in PANSS score with risperidone, compared with 36\% with olanzapine. An 8-week study randomly allocated patients to quetiapine (400-800 mg/day), olanzapine (10-20 mg/day) or risperidone (4-8 mg/day). The quetiapine group had a $31 \%$ improvement in PANSS score, while the olanzapine group had a $25 \%$ improvement and the risperidone group a $21 \%$ improvement. Weight gain was more prominent with olanzapine: $36 \%$ of the patients had a weight gain of $>5 \%$ from baseline, compared with $17 \%$ of the risperidone patients and
$13 \%$ of the quetiapine patients (Sachetti et al, 2003). The present study had similar results.

\section{References}

Allison, D. B., Mentore, J. L., Heo, M., et al (1999) Antipsychotic-induced weight gain: a comprehensive research synthesis. American Journal of Psychiatry, 156, 1686-1696.

American Diabetes Association (2004) Consensus development conference on antipsychotic drugs and obesity and diabetes. Diabetes Care, 27, 596-601.

Breier, A., Berg, P. H., Thakore, J. H., et al (2005) Olanzapine versus ziprasidone: results of a 28-week double-blind study in patients with schizophrenia. American Journal of Psychiatry, 162, 1879.

Bryden, K. E. \& Kopala, L. C. (1999) Body mass index increase of $58 \%$ associated with olanzapine. American Journal of Psychiatry, 156, 1835-1836.

Gupta, S., Masand, P. S. \& Virk, S. (2004) Weight decline in patients switching from olanzapine to quetiapine. Schizophrenia Research, 70, 57-62.

Lindenmayer, J. P., Czobor, P., Volavka, J., et al (2003) Changes in glucose and cholesterol levels in patients with schizophrenia treated with typical or atypical antipsychotics. American Journal of Psychiatry, 160, 290-296.

Sachetti, E., Valsecchi, P. \& Regeni, C. (2003) Comparison of quetiapine, olanzapine and risperidone in patients with schizophrenia: interim results of a randomised, rater-blinded study. European Neuropsychopharmacology, 13, S350.

Straker, D., Correll, C. U. \& Kramer-Ginsberg, E. (2005) Cost effective screening for the metabolic syndrome in patients treated with second generation antipsychotic medication. American Journal of Psychiatry, $162,1217-1221$.

Svesta, J., Synek, O. \& Zourkova, A. (2003) A double-blind comparison of olanzapine and quetiapine in the treatment of acute exacerbations of schizophrenic disorders. European Neuropsychopharmacology, 13, S291.

Taylor, D. M. \& McAskill, R. (2000) Atypical antipsychotics and weight gain - a systematic review. Acta Psychiatrica Scandinavica, 101, 416-432.

Tran, P. V., Hamilton, S. H. \& Kuntz, A. J. (1997) Double blind comparison of olanzapine versus risperidone in the treatment of schizophrenia and other psychotic disorders. Journal of Clinical Psychopharmacology, $17,407-418$.

\section{Use of herbal medications among out-patients in a psychiatry clinic in Sri Lanka}

\section{K. A. L. A. Kuruppuarachchi ${ }^{1}$ and A. Hapangama²}

1Professor of Psychiatry, Faculty of Medicine, University of Kelaniya, Ragama, Sri Lanka, email lalithkuruppu@lycos.com ${ }^{2}$ Lecturer in Psychiatry, Faculty of Medicine, University of Kelaniya, Ragama, Sri Lanka

\begin{abstract}
The use of complementary and alternative medicine is increasing among psychiatric populations. Herbal medications are a form of alternative and complementary medicine whose use has increased over the last decade. Two studies done among Western out-patient groups have reported rates of use of herbal medicine at $15 \%$ ('current use'; Matthews et al, 2003) and 24\% (Knaudt et al, 1999).

Eastern cultures have a long tradition of using herbal medicines for a variety of conditions and people on the Indian subcontinent, for example, seem to be very familiar with
\end{abstract}

them. Concomitant use of herbal and Western medications is commonly observed even in the West. Herbal preparations contain biologically active components, with unique sideeffect profiles (Matthews et al, 2003). There have been reports of clinically significant interactions between herbs and Western medications (Fugh-Berman, 2000). In a US study of patients who used alternative therapies, only $38.5 \%$ had discussed this with their physician (Eisenberg et al, 1998).

However, there is limited information available on the prevalence of use of herbal medications, the types of plants 